\title{
A Model of Income Evaluation: Income Comparison on Subjective Reference Income Distribution
}

\author{
Atsushi Ishida (Kwansei Gakuin University)*
}

July 3, 2021

\begin{abstract}
People's evaluation of the relative position of their income is not as accurate as the relative income hypothesis assumes. It is observed from empirical survey data that income evaluation is concentrated in the middle. We develop a model that assumes income comparison on a subjective income reference distribution to explain the centralization phenomenon of income evaluation. We conduct theoretical analysis and empirical parameter estimation using Bayesian statistical modeling. The theoretical analysis shows that the centralization of income evaluation distribution occurs when the subjective reference distribution is more dispersed than the objective distribution. Empirical analysis using Japanese data from 2015 shows that the relationship between subjective and objective distributions differed depending on social categories with different social experiences. Women had a more ambiguous distribution than men. Among men, those aged 45-54 had a subjective distribution closest to the objective distribution. Thus, the subjective reference income distributions that potentially define people's evaluation of their income and their differences based on social category were only clarified by constructing the model.
\end{abstract}

Keywords: income distribution; relative income hypothesis; Bayesian statistical modeling

\section{Introduction}

This article focuses on individuals' evaluations of their own income in relation to others within the income distribution. Income evaluation can be both the basis for satisfaction or dissatisfaction in terms of one's economic situation. It can also create a sense of fairness or unfairness regarding income distribution, which could lead to social change or stabilization. It is assumed that evaluation of income would be obtained by recognizing the relative position of one's own income in the distribution through comparison with others. Hence, this is also related to the empirical validity of the "relative income hypothesis" in economics.

The relative income hypothesis was first explicitly proposed by Duesenberry (1949). Duesenberry proposed the idea that an individual's consumption function depends on others' income and the relative position among them. He

*aishida@kwansei.ac.jp 
derived the theorem that "for any given relative income distribution, the percentage of income saved by a family will tend to be unique, invariant, and increasing function of its percentile position in the income distribution. The percentage saved will be independent of the absolute level of income. It follows that the aggregate saving ratio will be independent of the absolute level of income"(Duesenberry, 1949, 3). Duesenberry's idea has recently been reconsidered, especially in the field of subjective well-being studies. For example, the well-known Easterlin Paradox was proposed (Easterlin, 1974, 1995, 2005); it was empirically derived from longitudinal data in the United States, Japan, and European nations. It states that the average happiness of citizens remains constant over time, despite a sharp increase in national income per capita. The relative income hypothesis is a prominent supposition, explaining this type of paradox (Clark et al., 2008). Furthermore, there have been several empirical survey data analytical studies using relative income variables as explanatory variables (Clark and Oswald, 1996; Mcbride, 2001; Ferrer-i Carbonell, 2005; Senik, 2008; Clark and Senik, 2010).

As seen in Dusenberry's theorem, the relative income hypothesis generally assumes that people correctly perceive their relative position in the income distribution. This study focuses on the empirical validity of this assumption. As empirical evidence, we present survey data conducted in Japan in 2015 (see Section 4 for details of the data). Figure 1 shows the distribution of individual annual income ${ }^{1}$, and Figure 2 shows the distribution of relative evaluation of income, which ranges from 1 (lowest) to 10 (highest) ${ }^{2}$. Figure 1 shows the income distribution is well fitted by a lognormal distribution as theoretically expected (Hamada, 2003, 2004). As for theoretical expectation of the distribution of income evaluation, if people perceive their income levels correctly, the distribution is expected to be uniform as will be explained in the next section. However, Figure 2 shows concentration slightly below the middle point and is far from a uniform distribution.

[Figure 1 about here.]

[Figure 2 about here.]

These findings lead us to the puzzle of this article which is why the distribution of the relative evaluation of income level is centralized. The centralization of the distribution of class identification, which is a multidimensional evaluation of status, is well known. Several mathematical models have been proposed to explain this phenomenon (Fararo and Kosaka, 2003; Ishida, 2018). However, to the best of our knowledge, there are no studies on the mechanism of centralization in unidimensional income evaluation. To solve this puzzle, in the following sections, we introduce a model that assumes that comparisons are made on a subjective reference distribution of income that is shared among members of a group rather than the objective income distribution per se.

We first introduce a general model of income evaluation and derive some implications of the model. We then introduce a model imposing the assumption of

\footnotetext{
${ }^{1}$ The unit of income is the Japanese yen. We treated 344 cases with no individual income and three cases with 100 million yen as missing.

${ }^{2}$ The question in the questionnaire was as follows: Thinking about present-day Japanese society, how would you rate your own level of income below on a scale of 1 (the highest level) to 10 (the lowest level)?
} 
lognormal distribution as the theoretical distribution of income. Next, we conduct an empirical data analysis of Japanese survey data by employing Bayesian statistical modeling, based on the lognormal distribution model. Finally, we present the conclusions.

\section{General Model}

In this section, we introduce the model in a general form without specifying the types of income distribution and subjective reference income distribution. We then derive some implications.

\subsection{Model Assumption}

Let $Y \in\{0, \cdots, m\}$ be a discrete random variable of response to the $m+1$ scaled income evaluation question, which ranges from 0 (lowest) to $m$ (highest). Let $x \in(0, \infty)$ be the income level of an individual and $p$ be the probability that $x$ exceeds the income level $z$, that is, $p=\operatorname{Pr}(x \geq z)$.

We assume that an individual evaluates their relative position of income by repeatedly comparing themselves with others whom they randomly encounter on a subjective reference income distribution. It is further assumed that the subjective reference distribution reflects the biased pattern of an individual's daily encounters and/or their expectations about the distribution that are not based on their experience but on media information or rumors. Here, we make a baseline assumption that the subjective reference income distribution is identical and commonly shared among members of a social category, presupposing that their social experiences and perceptions are almost similar. In addition, when evaluating their income, the individual is assumed to respond according to the number of times they have outperformed others in the last $m$ comparisons.

The general model can be composed as

$$
\begin{aligned}
Y & \sim \operatorname{Binomial}(m, p), \\
p & =F_{s}(x), \\
x & \sim f_{o}(x),
\end{aligned}
$$

where $F_{s}$ is the cumulative distribution function (CDF in short) of the subjective reference income distribution and $f_{o}$ is the probability density function (PDF in short) of the objective income distribution.

We are ultimately interested in the distribution of $Y$. However, if $m$ is a constant, the distribution of $Y$ depends only on the parameter $p$ of the binomial distribution, so we are essentially interested in the distribution of $p$. Then, the PDF of $p$, denoted as $\varphi(p)$, is

$$
\begin{aligned}
\varphi(p) & =f_{o}(x) \frac{d x}{d p} \\
& =f_{o}(x) \frac{1}{\left\{F_{s}(x)\right\}^{\prime}}=\frac{f_{o}(x)}{f_{s}(x)} .
\end{aligned}
$$

From equation (2), $x$ can be expressed as the inverse CDF of the subjective reference distribution, i.e. $x=F_{s}^{-1}(p)$. Finally, we obtain the full form of the 
PDF of $p$ as

$$
\varphi(p)=\frac{f_{o}\left(F_{s}^{-1}(p)\right)}{f_{s}\left(F_{s}^{-1}(p)\right)} .
$$

By definition, we can confirm the following properties of $\varphi(p)$, which indicate that $\varphi(p)$ is surely a PDF:

$$
\begin{aligned}
\varphi(p) & =f_{o}(x) \frac{d x}{d p} \geq 0 \\
\int_{0}^{1} \varphi(p) d p & =\int_{0}^{1} f_{o}(x) \frac{d x}{d p} d p \\
& =\int_{-\infty}^{\infty} f_{o}(x) d x=1 .
\end{aligned}
$$

\subsection{Model Derivations}

Henceforth, we assume that $f_{o}, f_{s}$ is a unimodal and second-order differentiable PDF. Furthermore, we assume $F_{s}$, which is the CDF of $f_{s}$, is a strictly increasing function, and $F_{s}^{-1}$, which is the inverse CDF of $f_{s}$, is also a strictly increasing and differentiable function.

First, we assume, as a special case, that the subjective reference income distribution is equal to the objective income distribution, because there is no encounter bias. That is, $f_{o}=f_{s}$. Then, we obtain $\varphi(p)=1$, which means $p$ is uniformly distributed from 0 to 1 . That is, $p \sim \operatorname{Uniform}(0,1)$. This is an ideal situation of income evaluation, assumed by the relative income hypothesis, where people perceive the objective income distribution correctly and evaluate their income with respect to the exact relative position in the objective income distribution.

Now, we move on to more general situations where there is a difference between the objective income distribution and subjective reference income distribution, which is biased from the objective distribution. That is, $f_{o} \neq f_{s}$. Differentiating $\varphi(p)$, which is expressed as equation (4), we obtain the derivative of $\varphi(p)$ as

$$
\varphi^{\prime}(p)=\frac{f_{o}^{\prime}(x) f_{s}(x)-f_{o}(x) f_{s}^{\prime}(x)}{f_{s}(x)^{2}}\left\{F_{s}^{-1}(p)\right\}^{\prime} .
$$

Because $f_{s}(x)>0,\left\{F_{s}^{-1}(p)\right\}^{\prime}>0$ on their support from the assumption, the sign condition of $\varphi^{\prime}(p)$ solely depends on the relation between the magnitudes of $f_{o}^{\prime}(x) / f_{o}(x)$ and $f_{s}^{\prime}(x) / f_{s}(x)$ which are the growth rates of PDF of objective income distribution and subjective income distribution, respectively, that is:

$$
\varphi^{\prime}(p) \gtreqless 0 \Longleftrightarrow \frac{f_{o}^{\prime}(x)}{f_{o}(x)} \gtreqless \frac{f_{s}^{\prime}(x)}{f_{s}(x)} .
$$

Hence, if there is a point $x^{*}$ that makes the growth rates of both $f_{o}$ and $f_{s}$ equal, then the point $p^{*}=F_{s}\left(x^{*}\right)$ is a local maximum, or minimum, point of $\varphi(p)$. For a simple example, if $f_{o}^{\prime}\left(x^{*}\right)=f_{s}^{\prime}\left(x^{*}\right)=0$ which indicates both distributions have the same mode, then the point $p^{*}=F_{s}\left(x^{*}\right)$ yields $\varphi^{\prime}\left(p^{*}\right)=0$. 
We assume that there is a single point $p^{*}$ such as $\varphi^{\prime}\left(p^{*}\right)=0$. The second derivative at point $p^{*}$ is

$$
\varphi^{\prime \prime}\left(p^{*}\right)=\frac{f_{o}^{\prime \prime}\left(x^{*}\right) f_{s}\left(x^{*}\right)-f_{o}\left(x^{*}\right) f_{s}^{\prime \prime}\left(x^{*}\right)}{f_{s}\left(x^{*}\right)^{2}}\left(\left\{F_{s}^{-1}\left(p^{*}\right)\right\}^{\prime}\right)^{2} .
$$

The sign condition of $\varphi^{\prime \prime}\left(p^{*}\right)$ depends on the relation between the magnitudes of $f_{o}^{\prime \prime}(x) / f_{o}(x)$ and $f_{s}^{\prime \prime}(x) / f_{s}(x)$, which might be called the growth rate in terms of second derivative. That is,

$$
\varphi^{\prime \prime}\left(p^{*}\right) \gtreqless 0 \Longleftrightarrow \frac{f_{o}^{\prime \prime}(x)}{f_{o}(x)} \gtreqless \frac{f_{s}^{\prime \prime}(x)}{f_{s}(x)} .
$$

From these derivations, the condition for the appearance of the centralization effect in terms of income evaluation can be summarized as follows. There is a single maximum point that satisfies $f_{o}^{\prime}\left(x^{*}\right) / f_{o}\left(x^{*}\right)=f_{s}^{\prime}\left(x^{*}\right) / f_{s}\left(x^{*}\right)$ and $f_{o}^{\prime \prime}\left(x^{*}\right) / f_{o}\left(x^{*}\right)<f_{s}^{\prime \prime}\left(x^{*}\right) / f_{s}\left(x^{*}\right)$, and $p^{*}=F_{s}\left(x^{*}\right)$ is around 0.5 .

\section{Lognormal Distribution Model}

Next, we analyze a more restrictive model, in which both the objective and subjective reference income distributions are assumed to be lognormally distributed.

\subsection{Model Assumption}

Let $z \in(0, \infty)$ be an individual's income, and $x=\log z$ be the logged income ranging from $-\infty$ to $\infty$. We assume that income $z$ is lognormally distributed as a common assumption in the field of income distribution studies. Accordingly, $x$ is normally distributed. That is,

$$
\begin{aligned}
& z \sim \operatorname{Lognormal}(\mu, \sigma), \\
& x \sim \operatorname{Normal}(\mu, \sigma) .
\end{aligned}
$$

The PDF of the objective distribution of logged income $x$, which is a normal distribution with parameters $\mu_{o}, \sigma_{o}$, is denoted by $f_{o}\left(x \mid \mu_{o}, \sigma_{o}\right)$, and that of the subjective reference distribution, which is a normal distribution with parameters $\mu_{s}, \sigma_{s}$ is denoted by $f_{s}\left(x \mid \mu_{s}, \sigma_{s}\right)$. In concrete terms, the PDF can be expressed as

$$
f_{k}\left(x \mid \mu_{k}, \sigma_{k}\right)=\frac{1}{\sqrt{2 \pi} \sigma_{k}} \exp \left\{-\frac{\left(x-\mu_{k}\right)^{2}}{2 \sigma_{k}^{2}}\right\}, \quad(k=o, s)
$$

\subsection{Model Derivation}

Let us perform some derivation from the model.

First, to determine the condition of a local maximum point of the distribution $p$, we specify the growth rate of the objective and subjective reference income 
distribution ${ }^{3}$. The growth rate of $f_{k}$ is

$$
\frac{f_{k}^{\prime}(x)}{f_{k}(x)}=\frac{\mu_{k}-x}{\sigma_{k}^{2}}
$$

and the growth rate in terms of the second derivative is

$$
\frac{f_{k}^{\prime \prime}(x)}{f_{k}(x)}=\left(\frac{\mu_{k}-x}{\sigma_{k}^{2}}\right)^{2}-\frac{1}{\sigma_{k}^{2}} .
$$

From condition (6), the necessary condition for a local maximum, or minimum, point of $\varphi(p)$ is

$$
\varphi^{\prime}\left(p^{*}\right)=0 \Longleftrightarrow \frac{f_{o}^{\prime}\left(x^{*}\right)}{f_{o}(x)}=\frac{f_{s}^{\prime}\left(x^{*}\right)}{f_{s}\left(x^{*}\right)} \Longleftrightarrow \frac{\mu_{o}-x^{*}}{\sigma_{o}^{2}}=\frac{\mu_{s}-x^{*}}{\sigma_{s}^{2}},
$$

then, we get

$$
x^{*}=\frac{\mu_{s} \sigma_{o}^{2}-\mu_{o} \sigma_{s}^{2}}{\sigma_{o}^{2}-\sigma_{s}^{2}} \quad\left(\sigma_{o} \neq \sigma_{s}\right) .
$$

In this model, it can be assumed that the objective income distribution is given exogenously and is invariable over a focal period. On the other hand, the subjective reference distribution can be assumed to vary endogenously within the mechanism of the model, depending on patterns of encounters and/or people's expectations about the distribution.

To take this into account, we introduce new parameters, indicating the difference in the parameters of the subjective distribution from those of the objective distribution, that is, $\delta=\mu_{s}-\mu_{o}$ and $\sigma_{s}^{2}=\alpha \sigma_{o}^{2}$. Then, the equation of $x^{*}$ can be rewritten as

$$
x^{*}=\mu_{0}+\frac{\delta}{1-\alpha} \quad(\alpha \neq 1 \text { and } \alpha>0) .
$$

Moreover, the local maximum, or minimum, point $p^{*}$ is

$$
p^{*}=F_{s}\left(x^{*}\right)=\frac{1}{2}\left[1-\operatorname{Erf}\left(\frac{\alpha \delta}{\sqrt{2}\left(\alpha^{2}-1\right) \sigma_{o}}\right)\right] .
$$

Erf stands for the error function defined by $\operatorname{Erf}(x)=2 \Phi(x \sqrt{2})-1$, where $\Phi(x)$ is the $\mathrm{CDF}$ of the standard normal distribution (Feller, 1968, 179). As a special case, if $\delta=0$, in which both distributions have the same mean parameter (the same median in lognormal distributions), then $x^{*}=\mu_{s}, p^{*}=F_{s}\left(\mu_{s}\right)=1 / 2$.

\footnotetext{
${ }^{3}$ In general, the canonical form of exponential family of distribution can be expressed as

$$
f(x \mid \theta)=\exp (x b(\theta)+c(\theta)+d(x))
$$
}

where $b(\theta)$ is called a natural parameter (Dobson and Barnett, 2008, 46). Then, the growth rate and that in terms of the second derivative of $f$ would be

$$
\begin{aligned}
\frac{f^{\prime}(x \mid \theta)}{f(x \mid \theta)} & =b(\theta)+d^{\prime}(x), \\
\frac{f^{\prime \prime}(x \mid \theta)}{f(x \mid \theta)} & =\left(b(\theta)+d^{\prime}(x)\right)+d^{\prime \prime}(x) .
\end{aligned}
$$


As for change of $x^{*}$ according to parameters $\delta, \alpha$, we get

$$
\begin{aligned}
& \frac{\partial x^{*}}{\partial \delta}=\frac{1}{1-\alpha} \gtrless 0 \Longleftrightarrow \alpha \lessgtr 1, \\
& \frac{\partial x^{*}}{\partial \alpha}=\frac{\delta}{(1-\alpha)^{2}} \gtreqless 0 \Longleftrightarrow \delta \gtreqless 0 .
\end{aligned}
$$

Furthermore, the sufficient condition for local maximum, or minimum, point $p^{*}$ is

$$
\varphi^{\prime \prime}\left(p^{*}\right) \gtrless 0 \Longleftrightarrow \frac{f_{o}^{\prime \prime}\left(x^{*}\right)}{f_{o}(x)}-\frac{f_{s}^{\prime \prime}\left(x^{*}\right)}{f_{s}\left(x^{*}\right)}=\frac{1-\alpha}{\alpha \sigma_{o}^{2}} \gtrless 0 \Longleftrightarrow \alpha \lessgtr 1 .
$$

These results show that $p^{*}$ is the local maximum point when $\alpha>1$, that is, when the dispersion of the subjective distribution is greater than that of the objective distribution. They also show that the larger $\delta$ is, the smaller the maximum point of $p^{*}$ becomes.

Hence, the condition for the appearance of the centralization effect can be summarized as $\alpha>1$ and $\delta$ is around 0. Furthermore, if $g(\alpha)=(1-\alpha) /\left(\alpha \sigma_{o}^{2}\right)$, then $g^{\prime}(\alpha)=-\left(\alpha^{2} \sigma_{o}^{2}\right)^{-1}<0$, which indicates as $\alpha$ increases, the graph around the local maximum point become keener.

In summary, when the subjective income distribution, which is formed by biased encounters and/or people's expectations, is more widely dispersed than the actual objective income distribution, the distribution of income evaluation is more concentrated in the middle. Conversely, behind the middle-concentrated distribution of income evaluation, we can assume the existence of a subjective income distribution that is more dispersed than the objective distribution. In that sense, the subjective distribution is a blurred projection of the objective income distribution.

\subsection{Example of $p$ distribution}

In concrete terms, the PDF of $p, \varphi(p)$, assuming objective and subjective lognormal income distributions, can be expressed as

$$
\begin{aligned}
\varphi(p) & =\frac{\sigma_{s}}{\sigma_{o}} \exp \left\{\frac{1}{2}\left[\Phi^{-1}(p)^{2}-\frac{\left(\mu_{s}-\mu_{o}+\sigma_{s} \Phi^{-1}(p)\right)^{2}}{\sigma_{o}^{2}}\right]\right\} \\
& =\alpha \exp \left\{\frac{1}{2}\left[\Phi^{-1}(p)^{2}-\frac{\left(\delta+\alpha \sigma_{o} \Phi^{-1}(p)\right)^{2}}{\sigma_{o}^{2}}\right]\right\}
\end{aligned}
$$

where $\Phi^{-1}(p)$ is the inverse probit function.

Figure 3 shows an example of objective and subjective logged income distributions, with parameters $\mu_{o}=\mu_{s}=5, \sigma_{o}=1, \sigma_{s}=2$ and the corresponding PDF of $p$, which indicates a middle-concentrated distribution.

[Figure 3 about here.]

Figure 4 shows an example of the trajectory of $p^{*}$, the maximum, or minimum, point of $\varphi(p)$, according to $\delta$ and $\alpha$ with the parameter $\sigma_{o}=1$. When the dispersion of the subjective distribution is smaller than that of the objective distribution, that is, $\alpha<1, p^{*}$ of the minimum point increases as $\delta$ increases. Meanwhile, when the dispersion of the subjective distribution exceeds that of the objective distribution, that is, $\alpha>1, p^{*}$ of the maximum point decreases as $\delta$ increases. 
[Figure 4 about here.]

\section{Empirical Data Analysis}

In this section, using the SSP2015 data, which is a Japanese social survey data including income and income evaluation variables, we estimate the parameters of the lognormal distribution model using the Bayesian statistical modeling method and analyze the differences in the parameters by subgroup.

\subsection{Data}

The data used for the analysis are from the Stratification and Social Psychology Project Survey (SSP2015), which is a Japanese national sampling survey of class identity, social images, and other related attitudes toward social inequality and social stratification. The survey was conducted between January and June 2015. The sampling procedure was a three-stage stratified random sampling. The sampling list was the Japanese electoral roll and the basic resident registration. Questionnaires were distributed to 8,309 male and female participants aged 20-64 in 450 locations. The mode of data collection was face-to-face interviews with computer assistance (Computer Assisted Personal Interview, CAPI). Consequently, 3,573 valid responses were obtained. The survey was conducted with the respondents' informed consent, and anonymity was retained throughout the survey process. The official response rate was $43.03 \%$ (SSP Project, 2016).

\subsection{Variables}

As mentioned in the introduction, the main variables in the model are individual annual income (Figure 1) and the 10-scaled income evaluation (Figure 2). In addition, we introduce gender (male and female) and age cohort as covariates to examine the differences in the income evaluation within different social categories and the differences in the subjective income distributions across the categories assumed to be behind the evaluation. We created a nominal variable of age cohort, consisting of categories of 10-year duration between 25 and 64 years of age, i.e. 25-34, 35-44, 45-54, and 55-64.

\subsection{Common Subjective Distribution Model}

In the following, we construct the model as a Bayesian statistical model and estimate the parameters. First, as a baseline model, we analyze a common subjective distribution model in which all members of a society potentially have the same subjective distribution, regardless of their social category.

Given the data of income $Y[n]$ and income evaluation $X[n]$ for each individual $n(n=1,2, \cdots, N)$, we estimate the parameters $\mu_{s}$ and $\sigma_{s}$ of the subjective income distribution and the parameters $\mu_{o}$ and $\sigma_{o}$ of the objective income dis- 
tribution. The Bayesian model is as follows ${ }^{4}$ :

$$
\begin{aligned}
Y[n] & \sim \operatorname{Binomial}(9, p[n]) \\
p[n] & =F_{\text {Lognormal }}\left(X[n] \mid \mu_{s}, \sigma_{s}\right) \\
X[n] & \sim \operatorname{Lognormal}\left(\mu_{o}, \sigma_{o}\right) \\
\mu_{s} & \sim \operatorname{Uniform}(0,10) \\
\sigma_{s} & \sim \operatorname{Uniform}\left(0, \mu_{s}\right) \\
\mu_{o} & \sim \operatorname{Uniform}(0,10) \\
\sigma_{o} & \sim \operatorname{Uniform}\left(0, \mu_{o}\right)
\end{aligned}
$$

We employed Stan version 2.26.1 via the R package cmdstanr 0.4.0 (Stan Development Team, 2021) for Bayesian statistical inference with MCMC sampling. We conducted four chains of sampling for 6,000 iterations each, which included 1,000 initial iterations as warm-up samples. The thin interval was set to one to generate 20,000 sampled points of the posterior distribution.

\section{[Table 1 about here.]}

Table 1 summarizes the MCMC estimation of the posterior distributions for the common subjective distribution model. Because $\hat{R}$ of each parameter is approximately 1.000 , we can safely conclude that the MCMC sampling converged (Gelman et al., 2013, 284-6).

[Figure 5 about here.]

Figure 5 shows the estimated objective and subjective income distribution with posterior means as the representative estimated parameters. Table 1 and Figure 5 show that the subjective income distribution has a slightly larger mean and a much larger variance than the objective one. Consequently, we can say that the relationship between the subjective and objective income distributions produces a slightly positively skewed and centered income evaluation distribution.

\subsection{Different Subjective Distributions Model}

Next, we prepare a more complex and more realistic model with the additional assumption that each social category shares a different subjective income distribution, reflecting differences in social experience.

In this model, in addition to the assumption of the common model, we assume different parameters $\mu_{s}[k]$ and $\sigma_{s}[k]$ of the subjective income distribution and different parameters $\mu_{o}[k]$ and $\sigma_{o}[k]$ of the objective income distribution for each social category $k(k=1,2, \cdots, K)$. In the actual data analysis, social category $k$ consisted of a combination of gender and age cohorts, i.e. 1: male and 25-34, 2: male and 35-44, 3: male and 45-54, 4: male and 55-64, 5: female and 25-34, 6: female and 35-44, 7: female and 45-54, and 8: female and 55-64.

${ }^{4}$ The Stan code is appeared in the Appendix. 


$$
\begin{aligned}
Y[n] & \sim \operatorname{Binomial}(9, p[n]) \\
p[n] & =F_{\text {Lognormal }}\left(X[n] \mid \mu_{s}[k], \sigma_{s}[k]\right) \\
X[n] & \sim \operatorname{Lognormal}\left(\mu_{o}[k], \sigma_{o}[k]\right) \\
\mu_{s}[k] & \sim \operatorname{Uniform}(0,10) \\
\sigma_{s}[k] & \sim \operatorname{Uniform}\left(0, \mu_{s}[k]\right) \\
\mu_{o}[k] & \sim \operatorname{Uniform}(0,10) \\
\sigma_{o}[k] & \sim \operatorname{Uniform}\left(0, \mu_{o}[k]\right)
\end{aligned}
$$

Figure 6 and 7 show the individual income distribution and income evaluation distribution for each social category, respectively.

[Figure 6 about here.]

[Figure 7 about here.]

As in the previous model, we conducted four chains of Bayesian statistical sampling for 6,000 iterations, each including 1,000 warm-up samples with one thin interval to generate 20,000 sampled points of posterior distribution. The results of the MCMC estimation indicate that all parameter estimations converged properly ${ }^{5}$.

Figure 8 shows estimated parameters of objective and subjective income distribution for each social category represented by posterior mean and interval between 0.05 and 0.95 quantiles. Figure 9 shows the estimated objective and subjective logged income distributions for each social category.

[Figure 8 about here.]

[Figure 9 about here.]

First, women have a subjective income distribution with greater variability than men, suggesting that income evaluation is based on subjective experiences that are quite ambiguous. This may reflect the fact that women's participation in the labor market is still lower than that of men, and many women participate in the labor market as a marginal labor force in Japan. Second, among men, the difference in the subjective distribution from the objective distribution is smallest in the age cohort of 45-54 years. This suggests that middle-aged men, who are expected to be the breadwinners in Japan, are the most realistic in comparing incomes and evaluating incomes based on this comparison.

Finally, we measure the distance of subjective income distribution $f_{s}$ from the reference point of the objective income distribution $f_{o}$ by using the KullbackLeibler distance. The Kullback-Leibler distance from $f_{o}$ to $f_{s}$ can be defined as

$$
D\left(f_{o} \| f_{s}\right)=\int f_{o}(x) \log \frac{f_{o}(x)}{f_{s}(x)} d x .
$$

\footnotetext{
${ }^{5}$ The Stan code and the summary table of MCMC estimation are appeared in the Appendix.
} 
Under the assumption of lognormal income distribution, the parametric equation is obtained as

$$
D\left(f_{o} \| f_{s}\right)=\log \sigma_{s}-\log \sigma_{o}+\frac{\sigma_{o}^{2}+\left(\mu_{o}-\mu_{s}\right)^{2}}{2 \sigma_{s}^{2}}-\frac{1}{2} .
$$

Figure 10 shows the Kullback-Leibler distance from the objective income distribution to the subjective income distribution for each social category. Again, we can see that women have a subjective income distribution that is farther from the objective one than men, and among men, the 45-54 age cohort has a subjective distribution that is closest to reality.

[Figure 10 about here.]

\section{Conclusion}

Thus far, we have developed a model that assumes income comparison on a subjective income reference distribution to explain the centralization phenomenon of income evaluation and have conducted theoretical analysis and empirical parameter estimation using Bayesian statistical modeling.

The theoretical analysis shows that income evaluation centralization occurs when the subjective distribution is more dispersed than the objective distribution. Furthermore, in a specific model assuming a lognormal distribution, we could parametrically analyze the effect of the relationship between the subjective and objective distributions on the distribution of income evaluation.

Empirically, using Japanese data from 2015, we confirmed that the empirically observed centralization of income evaluation can be explained by assuming a subjective distribution with large variance and ambiguity. Furthermore, we found that the relationship between the subjective and objective distributions differed depending on gender and age cohorts with different social experiences. Specifically, women had a more ambiguous distribution than men, while among men, those aged 45-54 had a subjective distribution closest to the objective distribution.

Thus, the subjective reference income distributions that potentially define people's evaluation of their income and their differences according to social category were only clarified by building the model. These results provide further insight into the relative income hypothesis in economics, in that people are not always able to evaluate the position of their own distribution very accurately, and that how accurately they can evaluate their position may depend on their social position of the individual. This can lead to biased income assessments, which has important implications for understanding people's satisfaction in unequal societies.

\section{Appendix}

Listing 1: Stan code for the common model

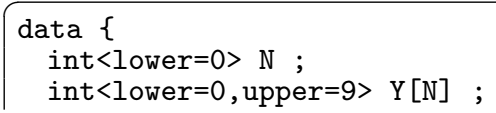




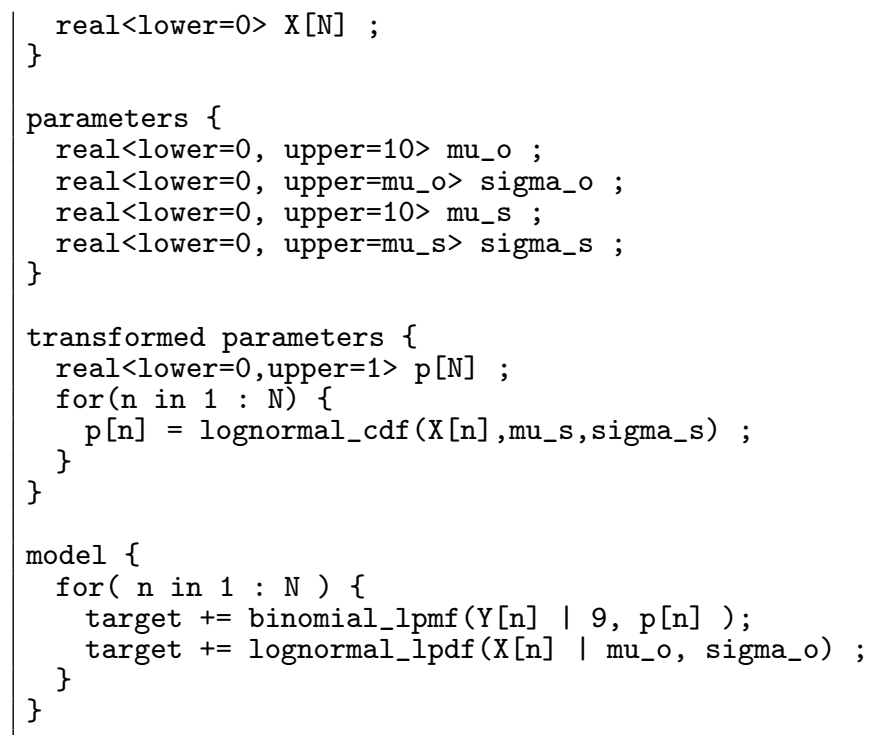

Listing 2: Stan code for the different model

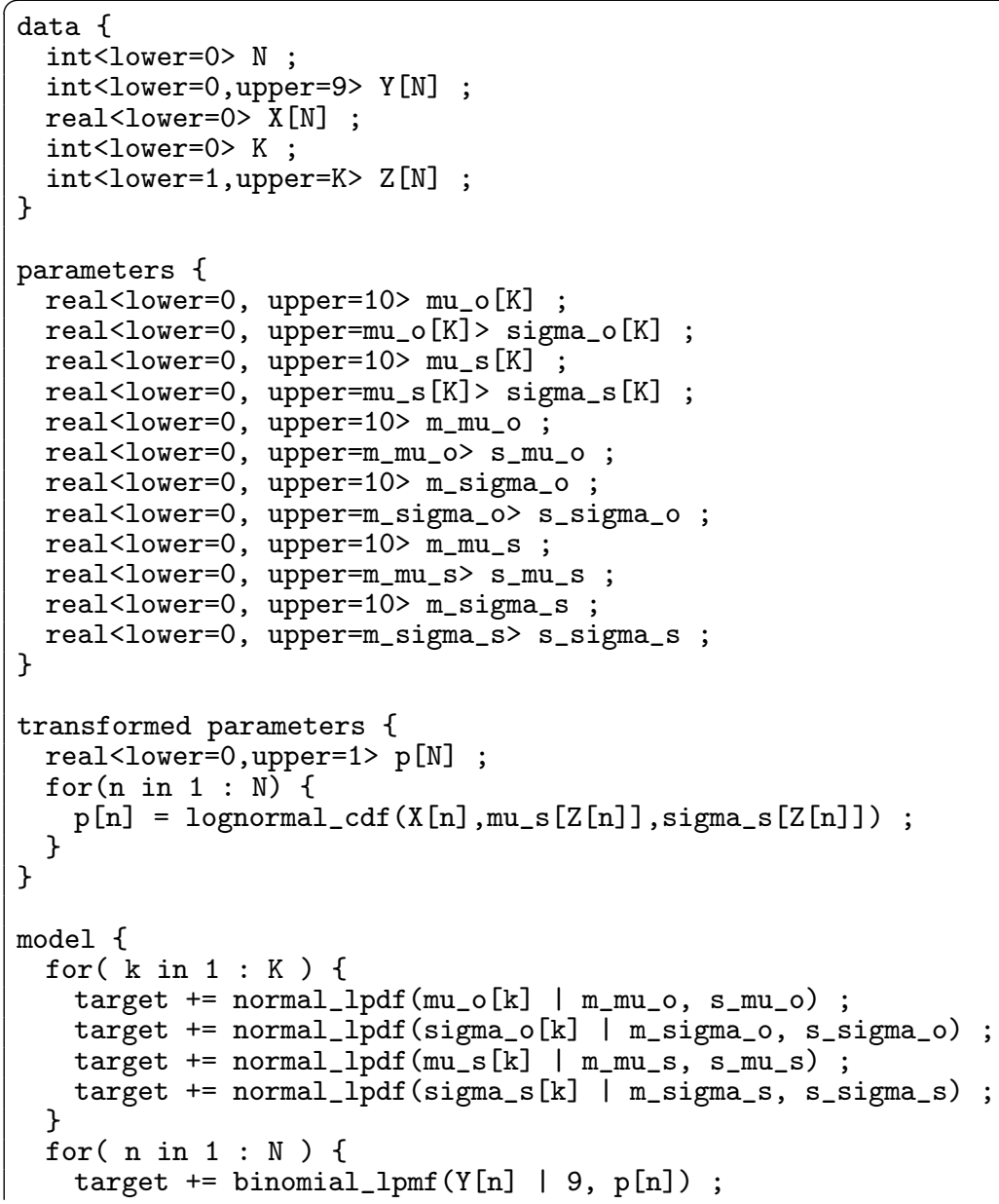


target $+=$ lognormal_lpdf $\left(\mathrm{X}[\mathrm{n}] \mid \mathrm{mu} \_\right.$o[Z[n] $]$, sigma_o[Z[n]] ) ;

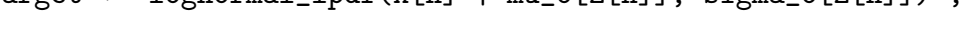

[Table 2 about here.]

\section{Acknowledgment}

We thank the SSP Project for permission to use the SSP2015 survey. This work was supported by JSPS KAKENHI Grant Numbers JP19H00609 and JP19K02065 as part of the SSP Project (http://ssp.hus.osaka-u.ac.jp/).

\section{References}

Clark, A. E., Frijters, P., and Shields, M. A. (2008). Relative Income, Happiness, Utility: An Explanation for the Easterlin Paradox and Other Puzzles. Journal of Economic Literature, 46(1):95-144.

Clark, A. E. and Oswald, A. J. (1996). Satisfaction and Comparison Income. Journal of Public Economics, 61(3):359-381.

Clark, A. E. and Senik, C. (2010). Who Compares to Whom? The Anatomy of Income Comparisons in Europe. Economic Journal, 120:573-594.

Dobson, A. J. and Barnett, A. G. (2008). An Introduction to Generalized Linear Models, 3rd ed. CRC Press, New York.

Duesenberry, J. S. (1949). Income, Saving, and the Theory of Consumer Behavior. Harvard University Press, Cambridge.

Easterlin, R. A. (1974). Does Empirical Growth Improve the Human Lot? Some Empirical Evidence. In David, P. A. and Reder, M. W., editors, Nations and Households in Economic Growth: Essays in Honor of Moses Abramovitz, pages 89-125. Academic Press, New York, NY.

Easterlin, R. A. (1995). Will Raising the Incomes of All Increase the Happiness of All? Journal of Economic Behavior \&3 Organization, 27(1):35-47.

Easterlin, R. A. (2005). Feeding the Illusion of Growth and Happiness: A Reply to Hagerty and Veenhoven. Social Indicators Research, 74(3):429-443.

Fararo, T. J. and Kosaka, K. (2003). Generating Images of Stratification: A Formal Theory. Kluwer Academic Publisher, Dordrecht, Netherlands.

Feller, W. (1968). An Introduction to Probability Theory and Its Applications, Vol. 1, 3rd ed. John Wiley \& Sons, New York.

Ferrer-i Carbonell, A. (2005). Income and Well-being: An Empirical Analysis of the Comparison Income Effect. Journal of Public Economics, 89(5-6):9971019 . 
Gelman, A., Carlin, J. B., Stern, H. S., David B. Dunson, D. B., Vehtari, A., and Rubin, D. B. (2013). Bayesian Data Analysis, 3rd Edition. Chapman \& Hall/CRC, Boca Raton.

Hamada, H. (2003). A generative model of income distribution. Journal of Mathematical Sociology, 27(4):279-299.

Hamada, H. (2004). Generative model of income distribution 2: Inequality of the iterated investment game. Journal of Mathematical Sociology, 28(1):1-24.

Ishida, A. (2018). Life-is-like-a-random-walk model of class identification. Sociological Theory and Methods, 33(2):261-276.

Mcbride, M. (2001). Relative-income Effects on Subjective Well-being in the Cross-section. Journal of Economic Behavior \& Organization, 45:251-278.

Senik, C. (2008). Ambition and Jealousy: Income Interactions in the "Old" Europe versus the "New" Europe and the United States. Economica, $75(299): 495-513$.

SSP Project, editor (2016). 2015nen Kaisou to Shakai Ishiki Zenkoku Chousa (Dai 1 kai SSP Chousa) Houkokusho /Research Report of the National Survey of Stratification and Social Psychology (the First SSP Survey)] (in Japanese). SSP Project.

Stan Development Team (2021). Stan modeling language users guide and reference manual, version 2.26 .

Vehtari, A., Gelman, A., Simpson, D., Carpenter, B., and Bürkner, P.-C. (2021). Rank-Normalization, Folding, and Localization: An Improved $\widehat{R}$ for Assessing Convergence of MCMC. Bayesian Analysis, pages $1-38$. 


\section{List of Tables}

1 Summary of MCMC estimation (common model) . . . . . . . . 16

2 Summary of MCMC estimation (different model) . . . . . . . 17 
Table 1: Summary of MCMC estimation (common model)

\begin{tabular}{|c|c|c|c|c|c|c|c|c|c|}
\hline & mean & median & $\mathrm{sd}$ & $\operatorname{mad}$ & q5 & q95 & rhat & ess_bulk & ess_tail \\
\hline$\mu_{o}$ & 5.497 & 5.497 & 0.020 & 0.020 & 5.465 & 5.529 & 1.000 & 18551.590 & 15634.409 \\
\hline$\sigma_{o}$ & 1.013 & 1.013 & 0.014 & 0.014 & 0.991 & 1.036 & 1.000 & 18169.652 & 14251.750 \\
\hline$\mu_{s}$ & 6.689 & 6.687 & 0.061 & 0.060 & 6.592 & 6.793 & 1.000 & 14202.602 & 13276.801 \\
\hline$\sigma_{s}$ & 4.740 & 4.732 & 0.190 & 0.188 & 4.439 & 5.065 & 1.001 & 13021.634 & 11365.716 \\
\hline
\end{tabular}

sd: standard deviation, mad: median absolute deviation, q5(95): 5(95) percentile of the distribution, rhat: Rhat convergence diagnostic, ess-bulk: bulk effective sample size, ess-tail: tail effective sample size estimate. For detail of rhat, ess-bulk, and esstail, see Vehtari et al. (2021). 
Table 2: Summary of MCMC estimation (different model)

\begin{tabular}{|c|c|c|c|c|c|c|c|c|c|}
\hline & mean & median & $\mathrm{sd}$ & $\operatorname{mad}$ & q5 & q95 & rhat & ess_bulk & ess_tail \\
\hline$\mu_{o}[1]$ & 5.730 & 5.730 & 0.044 & 0.044 & 5.657 & 5.802 & 1.000 & 27427.710 & 14672.491 \\
\hline$\mu_{o}[2]$ & 6.008 & 6.008 & 0.038 & 0.037 & 5.946 & 6.070 & 1.000 & 27148.328 & 14473.660 \\
\hline$\mu_{o}[3]$ & 6.229 & 6.229 & 0.038 & 0.039 & 6.166 & 6.291 & 1.000 & 29014.401 & 14074.282 \\
\hline$\mu_{o}[4]$ & 5.943 & 5.942 & 0.040 & 0.040 & 5.877 & 6.008 & 1.000 & 27714.120 & 15536.634 \\
\hline$\mu_{o}[5]$ & 4.950 & 4.950 & 0.068 & 0.067 & 4.838 & 5.064 & 1.000 & 29245.428 & 14868.744 \\
\hline$\mu_{o}[6]$ & 5.063 & 5.063 & 0.052 & 0.052 & 4.977 & 5.149 & 1.000 & 29649.919 & 14792.462 \\
\hline$\mu_{o}[7]$ & 5.203 & 5.203 & 0.053 & 0.053 & 5.115 & 5.289 & 1.001 & 30589.364 & 14729.750 \\
\hline$\mu_{o}[8]$ & 4.804 & 4.804 & 0.048 & 0.049 & 4.724 & 4.884 & 1.000 & 20907.677 & 15351.327 \\
\hline$\sigma_{o}[1]$ & 0.661 & 0.660 & 0.032 & 0.031 & 0.611 & 0.716 & 1.001 & 29976.439 & 15397.791 \\
\hline$\sigma_{o}[2]$ & 0.717 & 0.716 & 0.027 & 0.027 & 0.674 & 0.763 & 1.000 & 25776.291 & 14952.855 \\
\hline$\sigma_{o}[3]$ & 0.712 & 0.711 & 0.027 & 0.027 & 0.668 & 0.757 & 1.001 & 29229.363 & 14881.158 \\
\hline$\sigma_{o}[4]$ & 0.797 & 0.796 & 0.029 & 0.029 & 0.751 & 0.845 & 1.000 & 28930.399 & 14998.479 \\
\hline$\sigma_{o}[5]$ & 1.035 & 1.032 & 0.049 & 0.049 & 0.957 & 1.119 & 1.000 & 27350.269 & 14048.571 \\
\hline$\sigma_{o}[6]$ & 0.926 & 0.925 & 0.037 & 0.037 & 0.867 & 0.988 & 1.000 & 29697.588 & 14306.201 \\
\hline$\sigma_{o}[7]$ & 1.017 & 1.016 & 0.037 & 0.037 & 0.959 & 1.079 & 1.000 & 29278.940 & 16033.231 \\
\hline$\sigma_{o}[8]$ & 0.987 & 0.986 & 0.034 & 0.035 & 0.932 & 1.046 & 1.000 & 29143.387 & 15276.341 \\
\hline$\mu_{s}[1]$ & 6.490 & 6.477 & 0.129 & 0.121 & 6.306 & 6.719 & 1.000 & 15564.137 & 9261.695 \\
\hline$\mu_{s}[2]$ & 6.513 & 6.508 & 0.091 & 0.090 & 6.373 & 6.671 & 1.001 & 20507.393 & 12311.947 \\
\hline$\mu_{s}[3]$ & 6.450 & 6.448 & 0.050 & 0.049 & 6.370 & 6.534 & 1.000 & 25514.123 & 15071.909 \\
\hline$\mu_{s}[4]$ & 6.538 & 6.535 & 0.069 & 0.068 & 6.430 & 6.656 & 1.000 & 20161.515 & 13019.792 \\
\hline$\mu_{s}[5]$ & 6.857 & 6.843 & 0.283 & 0.295 & 6.413 & 7.345 & 1.000 & 13581.875 & 11935.472 \\
\hline$\mu_{s}[6]$ & 7.143 & 7.138 & 0.265 & 0.276 & 6.715 & 7.586 & 1.000 & 13789.686 & 13950.214 \\
\hline$\mu_{s}[7]$ & 6.950 & 6.948 & 0.193 & 0.194 & 6.631 & 7.268 & 1.000 & 20243.890 & 15002.906 \\
\hline$\mu_{s}[8]$ & 6.940 & 6.935 & 0.214 & 0.214 & 6.597 & 7.299 & 1.000 & 17924.467 & 14254.579 \\
\hline$\sigma_{s}[1]$ & 2.712 & 2.664 & 0.385 & 0.353 & 2.179 & 3.403 & 1.000 & 14310.356 & 9215.021 \\
\hline$\sigma_{s}[2]$ & 3.286 & 3.246 & 0.376 & 0.353 & 2.743 & 3.967 & 1.001 & 19441.870 & 12427.120 \\
\hline$\sigma_{s}[3]$ & 2.093 & 2.081 & 0.162 & 0.159 & 1.846 & 2.376 & 1.001 & 25121.623 & 15109.285 \\
\hline$\sigma_{s}[4]$ & 2.576 & 2.560 & 0.195 & 0.190 & 2.282 & 2.923 & 1.000 & 20745.280 & 13886.965 \\
\hline$\sigma_{s}[5]$ & 5.507 & 5.484 & 0.703 & 0.770 & 4.390 & 6.682 & 1.000 & 10827.131 & 8701.633 \\
\hline$\sigma_{s}[6]$ & 5.768 & 5.781 & 0.643 & 0.708 & 4.679 & 6.776 & 1.000 & 11429.765 & 11134.814 \\
\hline$\sigma_{s}[7]$ & 6.216 & 6.262 & 0.493 & 0.512 & 5.339 & 6.944 & 1.001 & 14695.803 & 13538.727 \\
\hline$\sigma_{s}[8]$ & 6.654 & 6.702 & 0.377 & 0.353 & 5.955 & 7.194 & 1.000 & 15949.725 & 13890.787 \\
\hline
\end{tabular}

sd: standard deviation, mad: median absolute deviation, q5(95): 5(95) percentile of the distribution, rhat: Rhat convergence diagnostic, ess-bulk: bulk effective sample size, ess-tail: tail effective sample size estimate. For detail of rhat, ess-bulk, and ess-tail, see Vehtari et al. (2021). 


\section{List of Figures}

1 Individual income distribution and fitted lognormal distribution (SSP 2015 data, unit: ten thousand Japanese yen) . . . . . . . . 19

2 Distribution of relative income evaluation (SSP 2015 data) . . . . 20

3 Example of objective and subjective income distributions $\left(\mu_{o}=\right.$ $\mu_{s}=5, \sigma_{o}=1, \sigma_{s}=2$ ) (left) and $p$ distribution (right) . . . . . 21

4 Example of trajectory of $p^{*}$ according to $\delta$ and $\alpha\left(\sigma_{o}=1\right) \ldots 22$

$5 \quad$ Estimated objective and subjective income distribution (left: logged income, right: income) . . . . . . . . . . . . . 23

6 Individual income distribution and fitted lognormal distribution for each social category . . . . . . . . . . . . . . . 24

$7 \quad$ Income evaluation distribution for each social category . . . . . . 25

8 Estimated parameters of objective and subjective income distribution for each social category (posterior mean and interval between 0.05 and 0.95 quantiles) . . . . . . . . . . . . . . . . . 26

9 Estimated objective and subjective logged income distribution for each social category . . . . . . . . . . . . . . . . 27

10 Kullback-Leibler distance from objective to subjective income distribution for each social category . . . . . . . . . . . . . . 28 


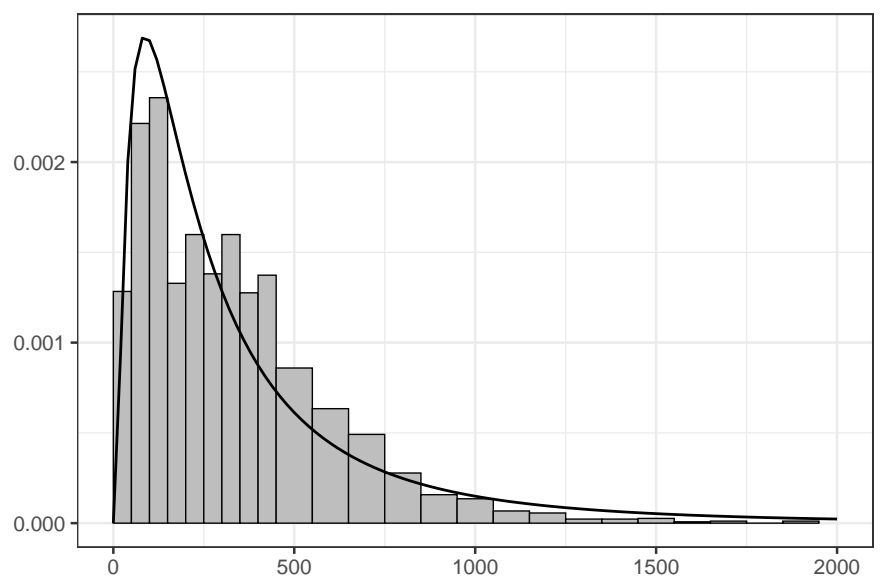

Figure 1: Individual income distribution and fitted lognormal distribution (SSP 2015 data, unit: ten thousand Japanese yen) 


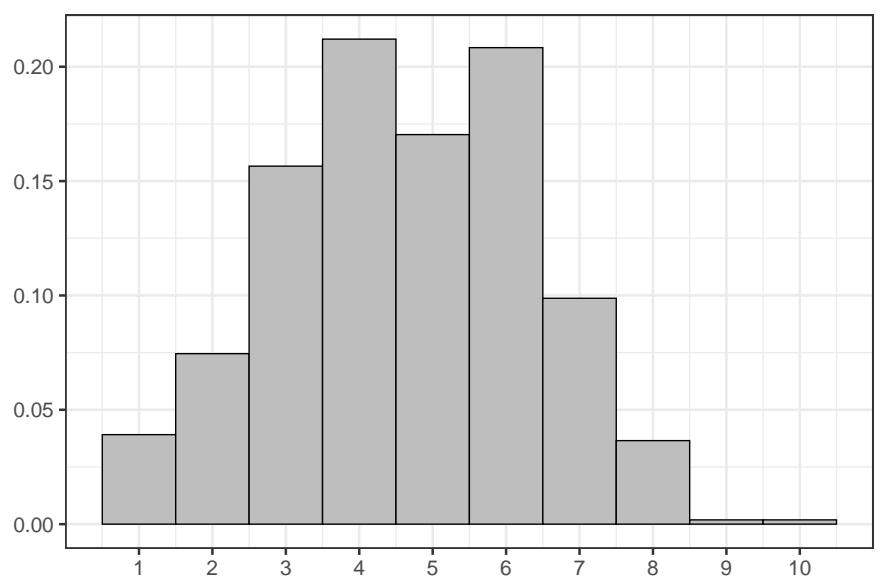

Figure 2: Distribution of relative income evaluation (SSP 2015 data) 

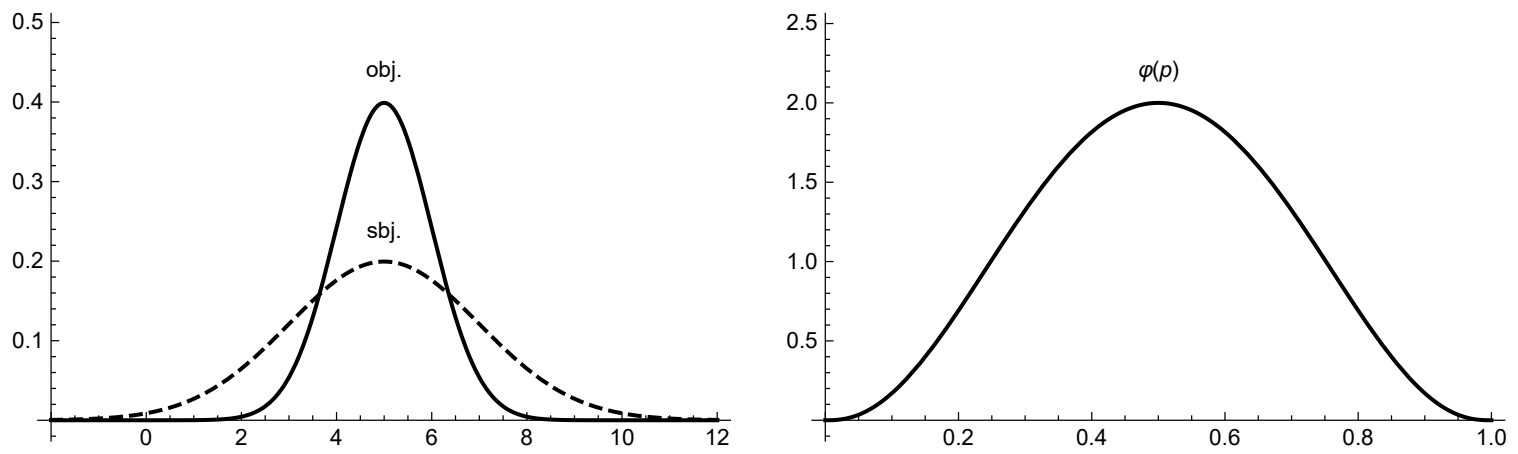

Figure 3: Example of objective and subjective income distributions $\left(\mu_{o}=\mu_{s}=\right.$ $5, \sigma_{o}=1, \sigma_{s}=2$ ) (left) and $p$ distribution (right) 


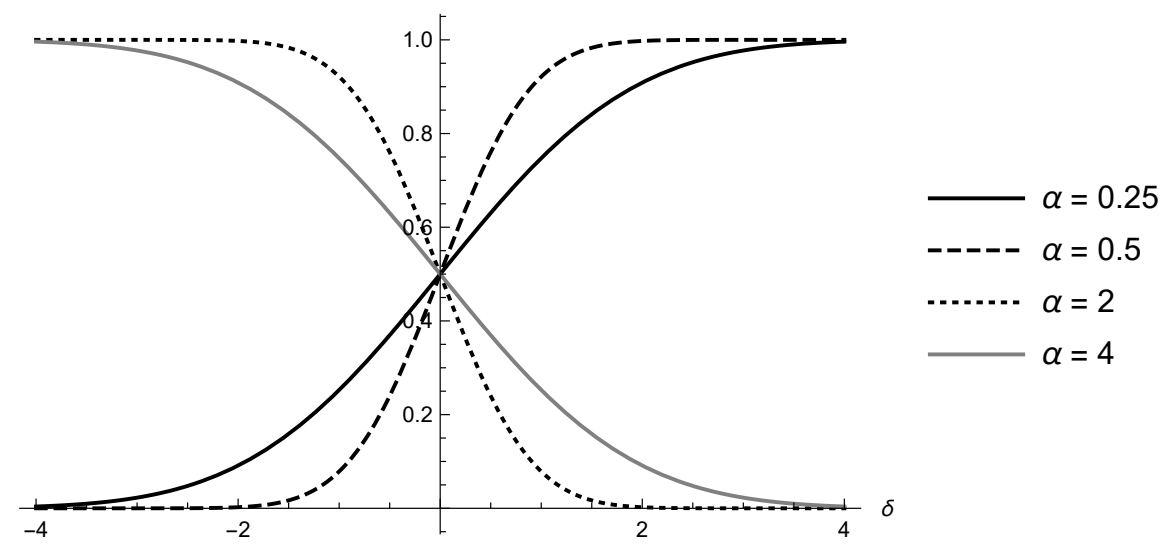

Figure 4: Example of trajectory of $p^{*}$ according to $\delta$ and $\alpha\left(\sigma_{o}=1\right)$ 

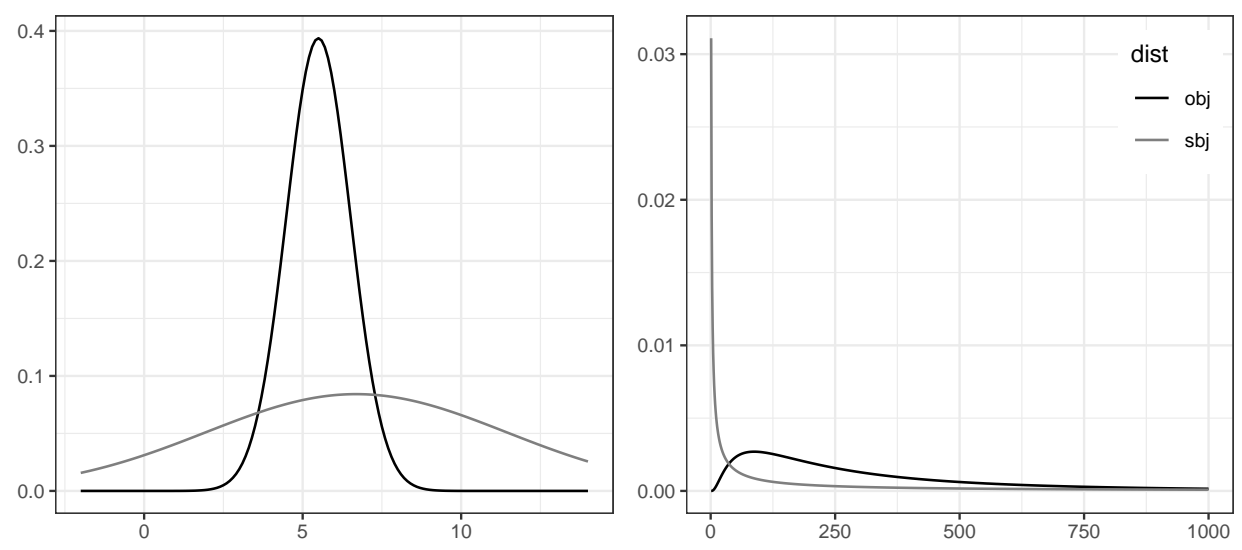

Figure 5: Estimated objective and subjective income distribution (left: logged income, right: income) 


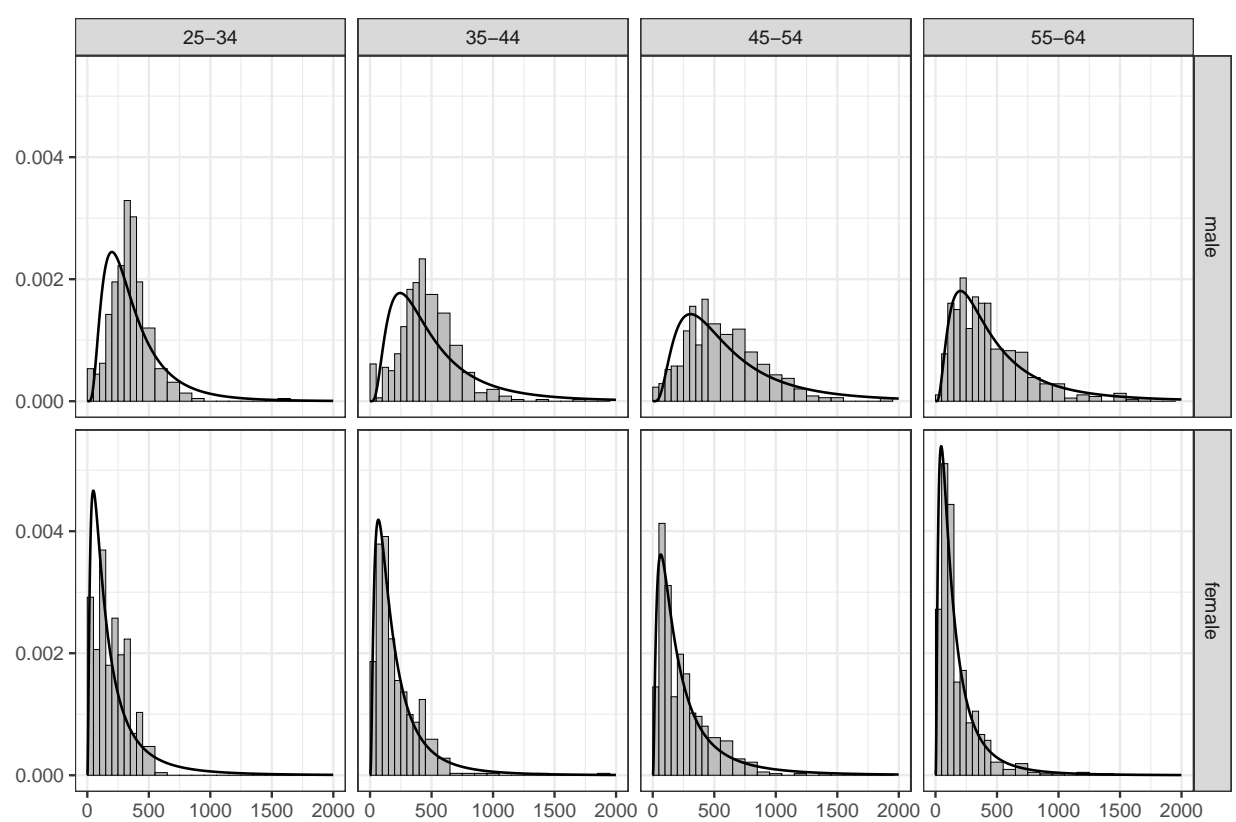

Figure 6: Individual income distribution and fitted lognormal distribution for each social category 


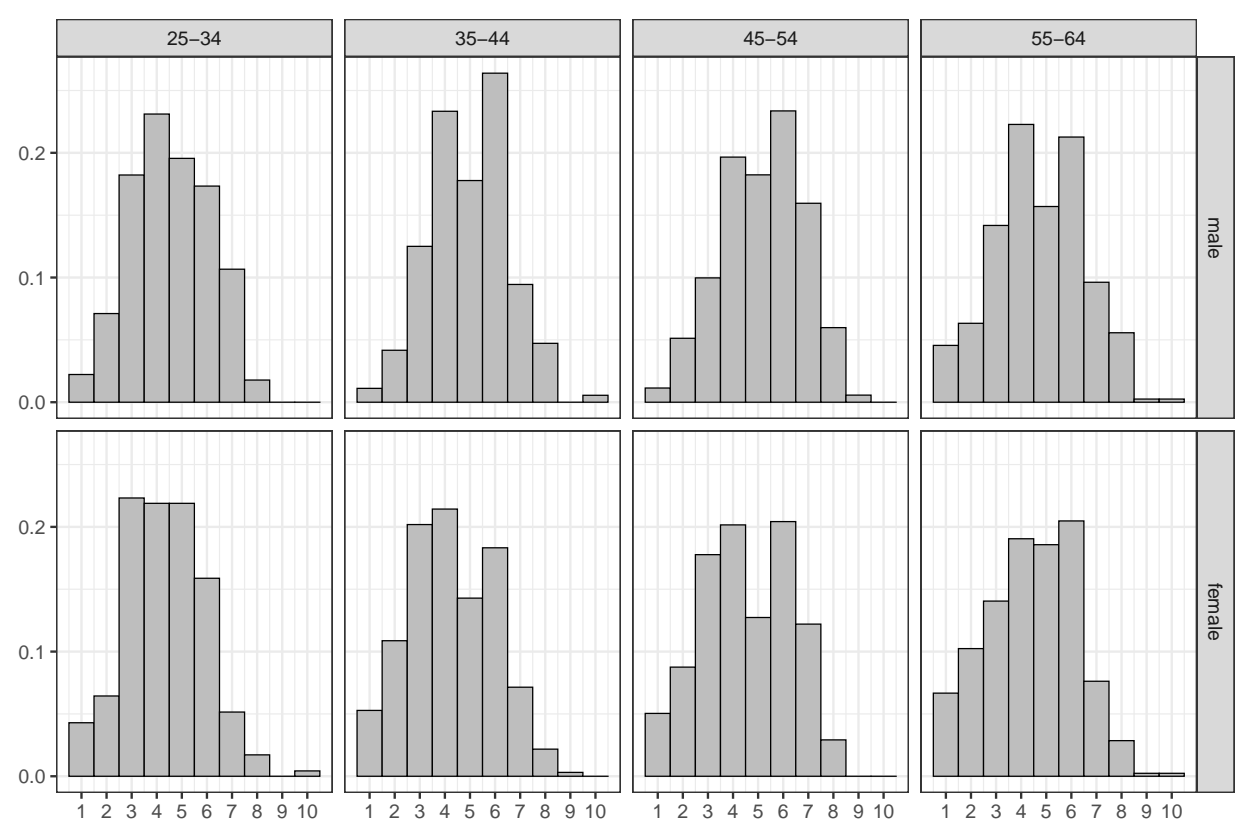

Figure 7: Income evaluation distribution for each social category 

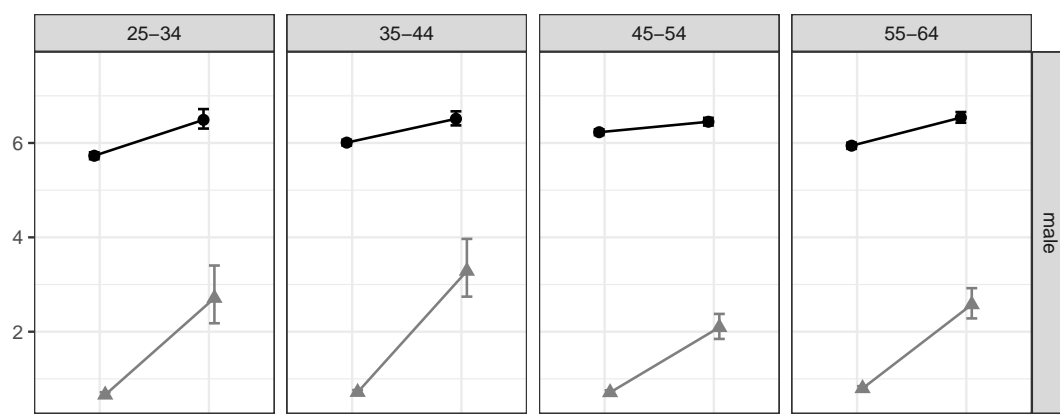

parameter
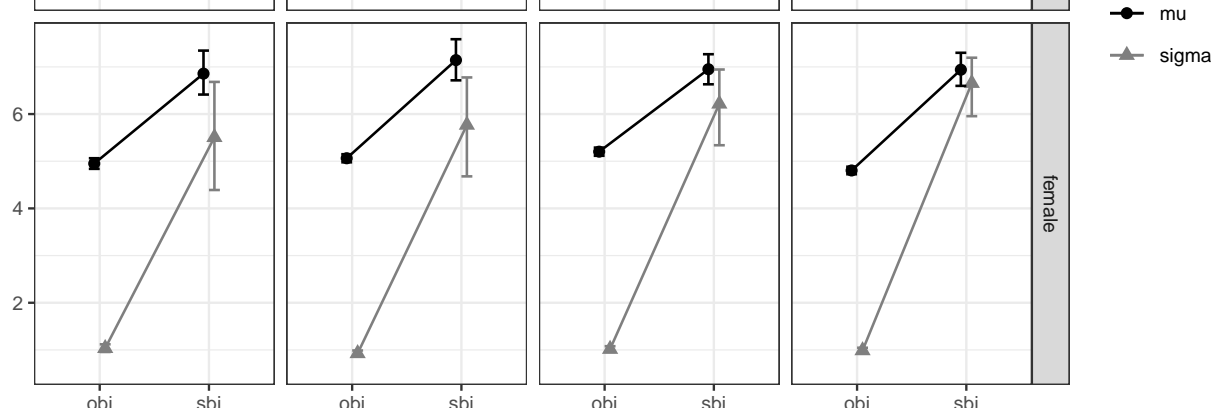

Figure 8: Estimated parameters of objective and subjective income distribution for each social category (posterior mean and interval between 0.05 and 0.95 quantiles) 


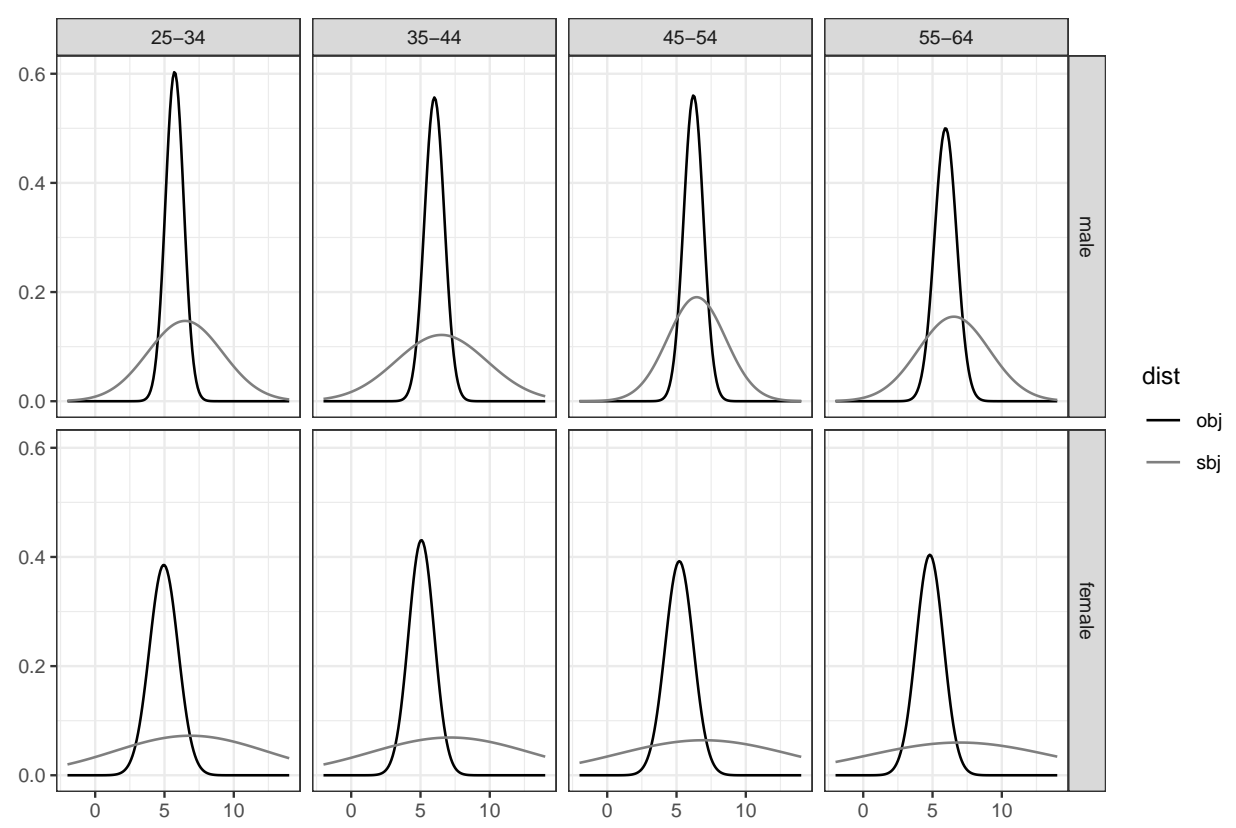

Figure 9: Estimated objective and subjective logged income distribution for each social category 


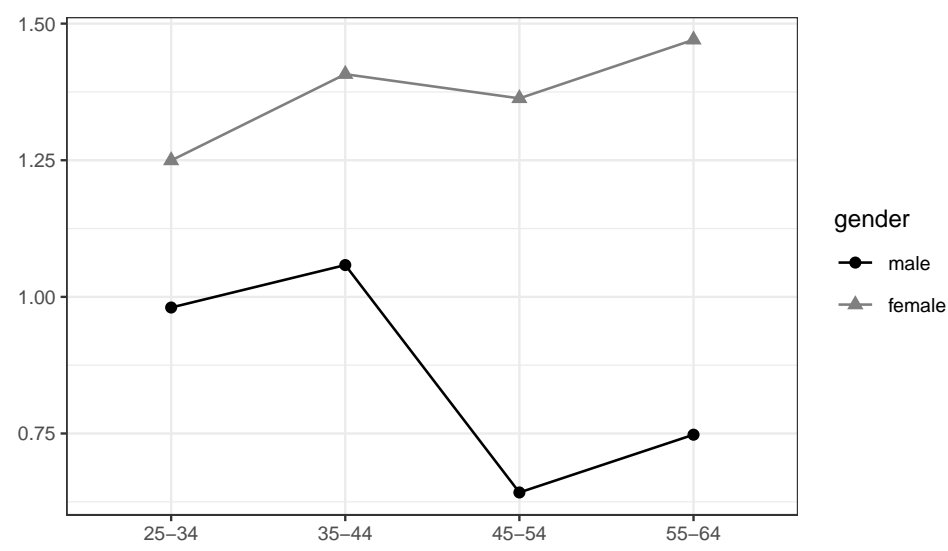

Figure 10: Kullback-Leibler distance from objective to subjective income distribution for each social category 